\title{
Role of Early Defibrillation in Success of Cardiopulmonary Resuscitation
}

\author{
El-Sayed Atiya', Monira Taha ${ }^{1 *}$, Gamela Nasr ${ }^{2}$, Abd El-fattah Frere ${ }^{3}$ \\ Departments of Emergency Medicine, Cardiology, Faculty of Medicine, Suez Canal University and Department of \\ Cardiology, Faculty of Medicine, Zagazig University
}

\begin{abstract}
Background: Despite the importance of early defibrillation in shockable cardiac arrest management that can affect the outcome, our Egyptian studies are very little in comparison with European and American updated studies. Aim: to improve Cardio pulmonary resuscitation protocols quality to give high rate of success of resuscitation protocols influencing ultimate outcome. Patients and Methods: Randomly selected 81 patients who are diagnosed as cardiac arrest either in hospital or out of hospital were included. They received advanced life support (ALS) protocol, and the effect of defibrillation time was assessed. Results: our study scope on the relation between early defibrillated shockable patients and time when first shock delivered, 25 patients received shock around 5 minutes (recorded by timer) from the beginning of resuscitation of confirmed cardiac arrest. The outcome as well as survival rate were the highest among patients who defibrillated early $(n=25)$. Of them, 23 patient got Return of Spontaneous Circulation (ROSC). However, among patients who defibrillated late (after 5 minutes, $n=35$ ), only 20 patients got ROSC. Conclusion: Our study showed that early defibrillation is the most important key to a successful outcome of inhospital patients with cardiac arrest who need defibrillation.
\end{abstract}

Keywords: cardiac arrest, shockable, automated defibrillator

\section{Introduction}

Cardiorespiratory arrest is failure of respiratory, cardiovascular, and neurological system according to Airway breathing, circulation or Disability problems. Recognizing the critically ill patient who will go to arrest is very important in prediction and prevention of cardiorespiratory arrest. Indicating the level of intervention by Early warning score $(\text { EWS })^{(1)}$. Heart rhythms associated with Cardiac arrest are divided into two groups: shockable rhythms (ventricular fibrillation/pulseless ventricular Tachycardia
(VF/VT) and non-shockable rhythms (asystole and pulseless electrical activity (PEA). The principle difference in the management of these two groups of arrhythmias is the need for defibrillation in patient with $\mathrm{VF} / \mathrm{VT}^{(2)}$. Subsequent actions, including chest compressions, airway management, ventilation, intravenous access, administration of adrenaline, identification and correction of reversible factors, are common on both groups ${ }^{(3)}$. The interventions that definitely improve survival after cardiac arrest are early defibrillation for VF/VT, and prompt, effective cardiopulmonary resusci-

*Corresponding Author: monirataha77@yahoo.com 
tation (CPR). Advanced airway intervention, and the delivery of drugs have not been shown to increase survival to hospital discharge after cardiac arrest but they are still included among Advanced Life Support (ALS) interventions Thus, during ALS, attention must be focused on early defibrillation and high quality, uninterrupted $\mathrm{CPR}^{(4)}$. Success is defined as termination of fibrillation or, more precisely the absence of VF/VT at 5 seconds after shock delivery. Although the ultimate goal is return of spontaneous circulation (ROSC) to achieve this, all defibrillation have 3 features in common; a power source capable of providing direct current, a capacitor that can be charged to a pre-determined energy level and two electrodes which are placed on the patient's chest through which the capacitor is discharged. Success depends on sufficient current being delivered to myocardium. Transthoracic impedance, shaving the chest, electrode size, coupling agents, Paddle force, electrode position, pads versus paddles, one shock versus three shock sequence all are factors affecting defibrillation success ${ }^{(5,6)}$. ROSC is an important phase in the continuum of resuscitation; however, the ultimate goal is to return the patient to a state of a normal cerebral function, a stable cardiac rhythm, and a normal hemodynamic function. Therefore, patients can leave hospital in a reasonable health condition with the minimum risk of other cardiac arrest. ALS in post resuscitation period influence the patient's ultimate outcome ${ }^{(7,8)}$. Despite the importance of early defibrillation in shockable cardiac arrest management, the Egyptian studies in this regard are very little.

\section{Patients and Methods}

This study was conducted as a descriptive, cross sectional, hospital based study at Intensive care unit and cardiac care unit in
Suez Canal university hospitals. Patients were recognized as cardiorespiratory arrest, either in hospital or outside of hospital. The sample populations were selected randomly from the patients arrived to the emergency department and admitted to hospital especially ICU and CCU (81 patients with cardiac arrest). Patients were recruited according to the following inclusion criteria; cardiac arrest (due to airway problem, breathing problem, circulation problems, or CNS problems), patient going to arrest (peri arrest) according to EWS. Exclusion criteria included death on arrival (DOA) and signs indicating death for long time as pooling of blood, rigor mortis, loss of cornea luster, severe crash brain trauma, end stage liver, renal, or cardiac diseases, a written request of the patient of "Do not attempt resuscitation (DNAR)", or patient $s^{\prime}$ relatives refusal to participate in the study. Complete medical history and clinical examination were carried out for all included patients.

\section{Results}

The mean age of the studied patients was $51.8 \pm 7.92$ years with most of the patients in age group 61-70 years (30.68\%). Most of the patients $(56.79 \%)$ were males. Shockable patients were divided into: i) rhythm: ventricular fibrillation in $39.5 \%$ of the patients and, ii) pulseless ventricular tachycardia in $34.57 \%$. In addition, non-shockable rythm were divided into: i) asystole (14.8\%) and ii) pulseless electrical activity (11.11\%). Table (1) classifies patients of shockable cardiac arrest according time into early before 5 minutes $(41.7 \%)$ and late after 5 minutes (58.3\%) according to time of DC exposure. Table (2) shows number of patients in whom (ROSC) occurs as we find that return of spontaneous circulation occurred in (92\%) of early defibrillated patients, in the other hand only $(57.1 \%)$ of late 
defibrillated patients. Table (3) shows patients get ROSC whom defibrillated early (around 5 minutes).In the first minute $6 \mathrm{pa}$ tients get ROSC after first shock delivered, 5 patients get ROSC after first shock delivered in the second minute, in the third minute 3 patients get ROSC after first shock and 2 after second DC. This depends on time obtain DC and 2 minutes of continuous CPR after delivery of each shock. Table (4) shows comparison between early and late defibrillation studied shockable arrested patients, ROSC appear in (92\%) and (80 $\%)$ discharge at home in early group, (57.1\%) get ROSC and only (8.5\%) survived in late group of defibrillated patients.

Table 1: Classification of defibrillation according to time

\begin{tabular}{|l|lc|}
\hline & $\mathrm{N}=60$ & $\%$ \\
\hline $\begin{array}{l}\text { Early defibrillation } \\
(\leq 5 \mathrm{~min})\end{array}$ & 25 & 41.7 \\
$\begin{array}{l}\text { Late defibrillation } \\
(>5 \mathrm{~min})\end{array}$ & 35 & 58.3 \\
\hline
\end{tabular}

Table 2: Return of Spontaneous Circulation (ROSC)

\begin{tabular}{|l|lc|}
\hline ROSC & No. & $\%$ \\
\hline Shockable & & \\
Early $(n=25)$ & 23 & 92 \\
Late $(n=35)$ & 20 & 57.1 \\
Non-shockable $(n=21)$ & 1 & 4.7 \\
\hline
\end{tabular}

\section{Discussion}

Cardiac arrest, the process where the mechanical pumping activity of the heart stops, represents the final step in a common pathway, which, without intervention, leads to death. Cardiorespi-ratory arrest can be caused by a primary airway breathing or cardiovascular problem ${ }^{(3)}$. Defibrillation is the passage across the myocardium of an electrical current of sufficient magnitude to depolarize a ritical mass of myocardium, and enable restoration of coordinated electrical activity.

Table 3: Patients with ROSC in relation to time and number of shocks in early defibrillated group

\begin{tabular}{|l|c|c|}
\hline Time (min) & $\begin{array}{c}\text { patient } \\
(\mathrm{n}=23)\end{array}$ & shocks \\
\hline $1^{\text {st }} \min$ & 6 & 1 \\
\hline $2^{\text {nd }} \min$ & 5 & 1 \\
\hline \multirow{2}{*}{$3^{\text {rd }} \min$} & 3 & 1 \\
\hline \multirow{2}{*}{$4^{\text {th }} \min$} & 2 & 2 \\
\hline \multirow{2}{*}{$5^{\text {th }} \min$} & 2 & 2 \\
\cline { 2 - 3 } & 1 & 3 \\
\cline { 2 - 3 } & 2 & 3 \\
\hline
\end{tabular}

Table 4: Early and late defibrillation in shockble patients

\begin{tabular}{|l|cccc|}
\hline & $\begin{array}{c}\text { Early } \\
(\mathrm{n}=25)\end{array}$ & $\begin{array}{c}\text { Late } \\
(\mathrm{n}=35)\end{array}$ & $\%$ \\
\hline Number & 25 & 100 & 35 & 100 \\
ROSC & 23 & 92 & 20 & $57.1 \%$ \\
$\begin{array}{l}\text { Discharge at } \\
\text { home }\end{array}$ & 20 & 80 & 3 & $8.5 \%$ \\
$\begin{array}{l}\text { Failed resusci- } \\
\text { tation }\end{array}$ & 2 & 8 & 15 & $42.8 \%$ \\
Bad outcome & 3 & 12 & 17 & $48.5 \%$ \\
\hline
\end{tabular}

Defibrillation is defined as the termination of fibrillation or, more precisely the absence of ventricular fibrillation/ ventricular tachycardia (VF/VT) at 5 second after shock delivery; however, the goal of attempted defibrillation is to restore spontaneous circulation $^{(5)}$. This study was conducted to describe the patterns of cardiac arrest in 81 arrested patients admitted to the cardiac care and intensive care unit at Suez Canal University Hospital and their outcome، focusing on the role of defibrillation in cardiopulmonary resuscitation. The mean age of the studied patients was $51.8 \pm 7.92$ years with most of the patients in age group 6170 years $(30.6 \%)$. This is consistent with Svensson et al, who studied 2160 in- 
hospital cardiac arrest records who were sent to CPR center and evaluated 612 patients needed defibrillation. Their average age was $57.1 \pm 21.2$ years ${ }^{(9)}$. Our study found that Most of the patients (56.79\%) were males and most of the patients (61.73\%) were from urban areas. This is consistent with a previous study in a national scheme for public access defibrillation in England and Wales ${ }^{(10)}$. In our study we classified victims of arrested patients according to arrest rhythm into non shockable $(n=21)$ and shockable $(\mathrm{n}=60)$ whom divided according to time obtained shocks into early group (<5 min) who got good outcome also high survival rate and late group who obtained DC after 5 minutes ( $>5 \mathrm{~min}$ ). Our study scoped on the relation between earlydefibrillated shockable patients, and time when first shock delivered. Twenty-five patients received shock around 5 minutes, and their outcome and survival rate was the highest at all. Among 25 patients who were defibrillated early, 23 patient (92\%) got ROSC,20 patients were discharged at home but among 35 patients DC was late after 5 minutes, 20 patients (57.1\%) got ROSC and only 3 of them were discharged at home, in contrast among 21 patients whom their resuscitation without shocks only one patient get ROSC then die after. This is in agreement with Pell et al, since their study showed that the patients with early defibrillation stayed in hospital for a short period, probably indicating a better outcome $^{(11)}$. Regarding the outcome of the patients in this study, $28.4 \%$ of patients have good outcome and were discharge at home most belong to early defibrillation group of shockable patients, $25.9 \%$ had bad outcome and die after time belong to late defibrillation, and non shockable groups. This is consistent with the study of Valenzuela et al, that showed a total of 2160 inhospital cardiac arrest, 283 patients (46.2\%) had ROSC after CPR and 50 patients (8.2\%) survived to discharge from hospital. $29 \%$ of patients remained under ICU care after having $\operatorname{ROSC}^{(12)}$. We found that survival until hospital discharge was inversely related to the number of shocks required, and this was similar to passage of time, less time means more survival as 6 patients got ROSC and survived after one shock after the first minute of cardiac arrest and 5 patients got ROSC after 2 minutes also after first shock but only one patient got ROSC and did not survive after 9 shocks after resuscitation of 20 minutes. This is in consistent with Colquhoun et al, as a datasheet was mailed to the fire defense headquarters throughout Japan, and returned data were analyzed for 614 cases with VF in relation to the time interval from receipt of emergency call to defibrillation. The study concluded that early resuscitated group had high survival. In spite of these findings, a marked delay to defibrillation (more than $13 \mathrm{~min}$ ) was observed in the majority $(79.5 \%)^{(13)}$. This study compared between early and late defibrillation studied shockable arrested patients, ROSC appeared in (92\%) and (80\%) were discharged at home in early group in contrast to $(57.1 \%)$ got ROSC and only (8.5\%) survived. This appears similarly consistent to Soar et al, who found that among 612 patients who needed defibrillation, 250 patients had early defibrillation, 190 of them survived and discharged from hospital ${ }^{(2)}$.

\section{Limitations of our study}

This study did not include every factor that might influence outcome of CPR such as knowledge or experience of physicians, nurses, BLS and medical personnel and the system for early detection of cardiac arrest.

\section{Conclusion}

The results of our study showed that early defibrillation is the most important key to a 
successful outcome of in-hospital patients with cardiac arrest who need defibrillation. Defibrillation has to be performed as early as possible before VF/VT changes into asystole and before irreversible damage of the vital organs.

\section{References}

1. Nolan JP, Nadkarni VM, Billi JE, Bossaert L, Boettiger BW, Chamberlain D. et al., Part 2: International collaboration in resuscitation science: 2010 International Consensus on Cardiopulmonary Resuscitation and Emergency Cardiovascular Care Science with Treatment Recommendations. Resuscitation 2010; 81:e26-e31.

2. Soar J, Mancini ME, Bhanji F, Billi JE, Dennett J, Finn J, Ma MH, Perkins GD, Rodgers DL, Hazinski MF, Jacobs I, Morley PT; Education, Implementation, and Teams Chapter Collaborators. 2010 International Consensus on Cardiopulmonary Resuscitation and Emergency Cardiovascular Care Science with Treatment Recommendations. Part 12: Education, Implementation, and Teams. Resuscitation 2010;81:e288-e330.

3. Nolan JP, Hazinski MF, Billi JE, Boettiger BW, Bossaert L, de Caen AR. et al., Part 1: Executive summary: 2010 International Consensus on Cardiopulmonary Resuscitation and Emergency Cardiovascular Care Science With Treatment Recommendations. Resuscitation 2010;81:e1-e25

4. Shuster M, Billi JE, Bossaert L, de Caen AR, Deakin CD, Eigel B. et al., International $\mathrm{Li}$ aison Committee on Resuscitation; American Heart Association. Part 4: Conflict of interest management before, during, and after the 2010 International Consensus Conference on Cardiopulmonary Resuscitation and Emergency Cardiovascular Care Science with Treatment Recommendations. Resuscitation 2010; 81:e41-e47.

5. Kitamura T, Iwami T, Kawamura T, Nagao K, Tanaka H, Nadkarni VM, Berg RA, Hiraide $A$; implementation working group for All-Japan Utstein Registry of the Fire and Disaster Management Agency. Con- ventional and chest-compression only cardiopulmonary resuscitation by bystanders for children who have out-of hospital cardiac arrests: a prospective, nationwide, population-based cohort study. Lancet 2010;375 (9723):1347-1354.

6. Kitamura T, Iwami T, Kawamura T, Nagao $\mathrm{K}$, Tanaka H, Hiraide A ; Implementation Working Group for the All-Japan Utstein Registry of the Fire and Disaster Management Agency. Nationwide public-access defibrillation in Japan. N Engl J Med 2010;362 (11):994-1004.

7. Deakin CD, Morrison LJ, Morley PT, Callaway CW, Kerber RE, Kronick SL. et al., Advanced Life Support Chapter Collaborators. Part 8: Advanced life support: 2010 International Consensus on Cardiopulmonary Resuscitation and Emergency Cardiovascular Care Science with Treatment Recommendations. Resuscitation 2010 ;81: e93-e169.

8. Rea TD, Fahrenbruch C, Culley L, Donohoe RT, Hambly C, Innes J, Bloomingdale M, Subido C, Romines S, Eisenberg MS. CPR with chest compression alone or with rescue breathing. N Engl J Med 2010;363 (5):423-433.

9. Svensson L, Bohm K, Castren $M$, Pettersson $\mathrm{H}$, Engerström L, Herlitz J, Rosenqvist M. Compression-only CPR or standard CPR in out-of-hospital cardiac arrest. N Engl J Med 2010; 363 (5):434-442.

10. Meaney PA, Nadkarni VM, Kern KB, Indik $\mathrm{JH}$, Halperin HR, Berg RA. Rhythms and outcomes of adult in-hospital cardiac arrest. Crit Care Med 2010;38 (1):101- 108.

11. Pell JP, Sirel JM, Marsden AK, Ford I, Walker NL, Cobbe SM. Presentation, management, and outcome of out of hospital cardiopulmonary arrest: comparison by underlying aetiology. Heart 2003;89 (8): 839842.

12. Valenzuela TD, Roe DJ, Nichol G, Clark LL, Spaite DW, Hardman RG. Outcomes of rapid defibrillation by security officers after cardiac arrest in casinos. N Engl Med 2000;343 (17):1206-1209.

13. Colquhoun MC, Chamberlain DA, Newcombe RG, Harris R, Harris S, Peel K, 
Davies CS, Boyle R. A national scheme for public access defibrillation in England and Wales: early results. Resuscitation 2008;78 (3):275-280. 